\title{
Application of the theory of separability of second-order differential equations
}

\author{
W.SARLET* ${ }^{*}$ F.CANTRIJN*† and E.MARTÍNEZ** \\ * Theoretical Mechanics Division, University of Gent \\ Krijgslaan 281, B-9000 Gent, Belgium \\ ** Departamento de Matemática Aplicada, Universidad de Zaragoza \\ E-50009 Zaragoza, Spain
}

Submitted by W.FUSHCHYCH

Received 24 August 1993

\begin{abstract}
A comprehensive application is presented of a recent theory concerning a geometric characterization of separable second-order differential equations. The main purpose of the paper is to illustrate how the practical algorithm developed from this theory effectively works, and what the significance is of the different conditions entering the separability theorem. These conditions are recalled in a coordinate representation, so as to make the paper sufficiently self-contained for practical computations.
\end{abstract}

\section{Introduction}

Contemporary work on qualitative aspects of dynamical systems is mostly designed for systems of first-order ordinary differential equations of relatively low dimension. There are of course good reasons for this: higher-order differential equations can always be recast in an equivalent first-order form, and the complexity of the qualitative behaviour of orbits rapidly becomes mathematically intractable when the dimension is too high. Besides, important results have been obtained, such as the centre manifold theorem, which justify the restriction to low dimension, at least for certain qualitative aspects of interest.

Copyright (C) 1994 by Mathematical Ukraina Publisher. All rights of reproduction in any form reserved.

\footnotetext{
${ }^{\dagger}$ Senior Research Associate at the National Fund for Scientific Research (Belgium).
} 
Many problems with a physical origin, however, are expressed by secondorder differential equations. Newtonian and Lagrangian equations of motion in classical mechanics are good examples in that respect, and when it concerns an interacting multiple particle system, for instance, the dimension is rightaway fairly high. Rewriting such a second-order system in the form of an equivalent first-order system somehow destroys part of the inherent structure of the problem. A natural question then is whether a sort of reduction of order could be achieved by searching for a suitable "reference frame" (i.e. a suitable coordinate system) with respect to which the given system of second-order equations at least partially decouples. Two recent papers $[3,5]$ deal with such questions, thereby making use of reasonably advanced differential geometric concepts. Kossowski and Thompson [3] are able to characterize a form of partial decoupling ("submersiveness") in terms of the existence of a distribution of vector fields with suitable properties. Existence results of this kind can of course be hard to verify in practice. Martínez et al [5] discuss the more restrictive case of complete decoupling into single second-order equations, but arrive at a characterization which, in principle, can be directly tested on any given system of second-order differential equations. In fact, the necessary and sufficient conditions for complete separability turn out to be of an algebraic and algorithmic nature. Certainly, they can be too tedious to compute by hand, but computer algebra packages nowadays are invaluable for adding a practical dimension to theoretical developments. For the history of the problem of full separability, we should also mention contributions by Ferrario et al (see e.g. $[1,2])$ for the special case of Lagrangian systems.

The purpose of the present paper is to put the theory in [5] to a hard test. The few examples of application of the theory which have been published so far ([5] and also [6]), were rather simple and straightforward illustrations. A hard case is needed to convince the public that the theory is really applicable, that all conditions play a role, and that the different subcases of the theoretical discussion really show up. Needless to say, complete separability will, generically speaking, be quite exceptional. Therefore, to have hope for success in analysing a concrete situation, there must be enough freedom left in the system we start from (i.e. enough arbitrary functions or free parameters). The three degrees of freedom system we will analyse in this paper has, perhaps a bit overambitiously, no less than 18 parameters for a start. The task then consists in identifying all regions in this parameter space where a full decoupling of the given system can be achieved.

For the remainder of this section, let us give an analytical formulation of the general problem under consideration, and introduce the main matrices and partial differential operators which play a key role in the computations.

Consider an arbitrary system of autonomous second-order equations in normal form

$$
\ddot{q}^{i}=f^{i}(q, \dot{q}), \quad i=1, \ldots, n .
$$


The problem of complete separability can then be formulated as follows: under what conditions for the given $f^{i}$ does there exist a regular coordinate transformation $Q^{j}=Q^{j}(q)$ such that in the new coordinates the system (1) takes the form

$$
\ddot{Q}^{j}=F^{j}\left(Q^{j}, \dot{Q}^{j}\right), \quad j=1, \ldots, n,
$$

and if such coordinates exist, how do we construct them?

Two sets of functions of coordinates $\left(q^{i}\right)$ and "velocities" $\left(v^{i}=\dot{q}^{i}\right)$ which are naturally associated to any second-order system like (1), are (the summation convention being used where appropriate),

$$
\Gamma_{j}^{i}(q, v)=-\frac{1}{2} \frac{\partial f^{i}}{\partial v^{j}}, \quad \Phi_{j}^{i}(q, v)=-\frac{\partial f^{i}}{\partial q^{j}}-\Gamma_{j}^{k} \Gamma_{k}^{i}-\Gamma\left(\Gamma_{j}^{i}\right),
$$

where $\Gamma=v^{i} \frac{\partial}{\partial q^{i}}+f^{i}(q, v) \frac{\partial}{\partial v^{i}}$ is the vector field related to (1). Geometrically speaking, the functions $\Gamma_{j}^{i}$ are the coefficients of a (nonlinear) connection, whereas $\left(\Phi_{j}^{i}\right)$ is truly a matrix representation of a linear endomorphism $\Phi$, a type $(1,1)$ tensor, which is called the Jacobi endomorphism corresponding to (1) (cf. [4]). Another important concept is the dynamical covariant derivative $\nabla$ : it is a derivation which is defined on tensorial objects of any type, and is type-preserving. Its action on functions is given by $\nabla g=\Gamma(g)$, whereas its action on $\Phi$, for example, creates a new type $(1,1)$ tensor whose matrix representation reads

$$
(\nabla \Phi)_{j}^{i}=\Gamma\left(\Phi_{j}^{i}\right)+\Gamma_{k}^{i} \Phi_{j}^{k}-\Phi_{k}^{i} \Gamma_{j}^{k}
$$

In order to have a feeling for the relevance of these concepts for the problem at hand, assume for a moment that the given equations (1) were already completely decoupled. Then we would have $\Gamma_{j}^{i}=0$ for $i \neq j$. Consequently, $\Phi$ would be diagonal and two of its diagonal elements could be equal only if they are constant. Finally, also $\nabla \Phi$ would be diagonal and, hence, commute with $\Phi$. This in turn means that the operator $\nabla$ preserves the eigenspaces of $\Phi$. Since we are talking here about intrinsically defined objects, we are actually looking at properties which will certainly be necessary for separability. They are, however, not quite sufficient. As a matter of fact, whenever $\Phi$ happens to be a constant multiple of the identity matrix, it is clear that $\Phi$ is not going to provide us with much information. In such a case, we have to appeal to another tensorial object for the characterization of separability, namely the so-called tension $\mathbf{t}$ related to the nonlinear connection $\left(\Gamma_{j}^{i}\right)$, given by

$$
\mathbf{t}_{j}^{i}=\Gamma_{j}^{i}-v^{k} \frac{\partial \Gamma_{j}^{i}}{\partial v^{k}}
$$

In the next section we will recall the main theorem about separability and comment on the algorithmic process for applications which follows from it. To keep the present paper reasonably self-contained, we will briefly sketch 
the meaning of other tensors and derivations entering the formulation of the theorem, referring to [5] for the full exposition and background of the theory. If one is only interested in doing the calculations, it will be sufficient to look at the coordinate expressions of all geometrical objects in question.

\section{Necessary and sufficient conditions for separability}

Let $U$ be an arbitrary type $(1,1)$ tensor with component matrix $\left(U_{j}^{i}(q, v)\right)$ and $X$ a vector with components $X^{k}(q, v)$. Here, and in the sequel, all indices run from 1 to $n$, with $n$ the dimension of $q$-space. We define a "vertical covariant derivative" $\mathrm{D}_{X}^{V}$ which acts in a non-trivial way on components of tensorial objects only and, for example, acting on $U$ produces a new type $(1,1)$ tensor $\mathrm{D}_{X}^{V} U$ with components

$$
\left(\mathrm{D}_{X}^{V} U\right)_{j}^{i}=X^{k} \frac{\partial U_{j}^{i}}{\partial v^{k}}
$$

Next, we associate to $U$ a type $(1,2)$ tensor $C_{U}^{V}$ whose action on arbitrary vectors $X$ and $Y$ is defined by

$$
C_{U}^{V}(X, Y)=\left[\mathrm{D}_{X}^{V} U, U\right](Y)
$$

the square brackets denoting the commutator of linear maps (i.e. of matrices).

Diagonalizability of a tensor, such as $U$, is a local and purely algebraic notion, which roughly means that the real Jordan normal form of the matrix $\left(U_{j}^{i}(q, v)\right)$ is diagonal, with eigenvalues that are smooth functions of $(q, v)$ in some appropriate domain. A final ingredient of the separability theorem below is the curvature tensor $R$ of the connection with coefficients $\Gamma_{j}^{i}$, defined by the second order system (1). This is a type $(1,2)$ tensor which is in fact derivable from the Jacobi endomorphism $\Phi$ via the property

$$
3 R(X, Y)=\mathrm{D}_{X}^{V} \Phi(Y)-\mathrm{D}_{Y}^{V} \Phi(X) .
$$

Theorem Given a second-order system (1). Assume that the Jacobi endomorphism $\Phi$ is diagonalizable and that the tensors $R, C_{\Phi}^{V}$ and $[\nabla \Phi, \Phi]$ are identically zero. Then, the system separates into individual second-order equations, one for each nondegenerate eigenvalue of $\Phi$, and into a multi-dimensional second-order system, separated from the rest, for each degenerate eigenvalue (which will then necessarily be constant). If for each such subsystem the corresponding tension $\mathbf{t}$ is diagonalizable and satisfies $C_{\mathbf{t}}^{V}=0$, then the given system completely decouples. Conversely, if (1) is completely separable, then all the previous conditions are verified.

Concerning the practical implementation of this result, a first comment is that the diagonalizability of $\Phi$, although it is the first condition mentioned in the theorem, will be the last one to impose in an application. Indeed, 
computing eigenvalues and the Jordan normal form of a matrix whose entries are functions of $2 n$ variables may be quite laborious and will certainly be simplified if one first takes into account the restrictions coming from the tensor equalities. To see how these equalities will work, let us first list the coordinate expressions of the tensors involved. As before, $\Gamma$ denotes the vector field representing the given second-order system. We then have, using (3), (5), (6) and (7):

$$
\begin{aligned}
R_{j k}^{i} & =\frac{1}{3}\left(\frac{\partial \Phi_{k}^{i}}{\partial v^{j}}-\frac{\partial \Phi_{j}^{i}}{\partial v^{k}}\right), \\
\left(C_{\Phi}^{V}\right)_{j k}^{i} & =\frac{\partial \Phi_{\ell}^{i}}{\partial v^{j}} \Phi_{l}^{l}-\Phi_{\ell}^{i} \frac{\partial \Phi_{k}^{\ell}}{\partial v^{j}}
\end{aligned}
$$

$$
[\nabla \Phi, \Phi]_{j}^{i}=\Gamma\left(\Phi_{k}^{i}\right) \Phi_{j}^{k}-\Phi_{k}^{i} \Gamma\left(\Phi_{j}^{k}\right)+\Gamma_{k}^{i} \Phi_{\ell}^{k} \Phi_{j}^{\ell}+\Phi_{k}^{i} \Phi_{\ell}^{k} \Gamma_{j}^{\ell}-2 \Phi_{k}^{i} \Gamma_{\ell}^{k} \Phi_{j}^{\ell} .
$$

Generally speaking the situation will be as follows. In most cases of interest, the right-hand sides $f^{i}$ of (1) will have a polynomial dependence on the velocity variables $v^{i}$ and assume, for example, that they contain a sufficient number of as yet undetermined parameters. The expressions $(8-10)$ then inherit a polynomial structure and since they must vanish identically, the coefficients of independent monomials in the $v^{i}$ must separately be put equal to zero. This will in general lead to quite severe restrictions on the parameters and resulting limitations on admissible $f^{i}$. It is advisable therefore to impose first the vanishing of (8), (9) and (10), in that order.

The calculations referred to above, are in principle rather straightforward, but can of course be quite messy as well. It is easy, however, to write procedures for computing $\Gamma_{j}^{i}, \Phi_{j}^{i}$ and the tensor components (8-10) in any computer algebra environment. We have made use of REDUCE to assist us in the tedious computations of the next sections. Splitting off coefficients of independent monomials is also a process which the computer is happy to do for us. It is not possible, however, to make the whole calculation run fully automatically. At each stage, different possible branches may show up which need a separate treatment, and other decisions may have to be taken which force us to keep the process interactive.

Once the algebraic equalities are satisfied, the hard problem (still of an algebraic nature) is to investigate the diagonalizability of $\Phi$. If $\Phi$ is diagonalizable with all eigenvalues different, then we are done: the theory guarantees complete separability. Moreover, the proof of the theorem also tells us how to find good coordinates to establish the decoupling. Indeed, the conditions of the theorem in particular imply that the eigenscpaces of $\Phi$ can be spanned by vectors depending on the coordinates $q^{i}$ only (this is in fact the content of the condition $C_{\Phi}^{V}=0$ ) and that they define complementary eigendistributions which are simultaneously integrable in the sense of Frobenius. In case all eigenvalues of $\Phi$ are nondegenerate, the eigenspaces are one-dimensional and 
good coordinates, in which the given system decouples, are obtained through the process of integrating these complementary one-dimensional distributions. The same procedure still applies if $\Phi$ is diagonalizable but has degenerate eigenvalues. However, the multi-dimensional subsystem corresponding to any of the constant degenerate eigenvalues may still be internally coupled. If then the conditions on the corresponding tension $\mathbf{t}$ are met, and if $\mathbf{t}$ has distinct eigenvalues, a decoupling transformation follows as before. If, however, $\mathbf{t}$ also has degenerate eigenvalues, a further coordinate transformation may be needed in order to achieve the final separation. The theory explains that in such a case the connection coefficients $\Gamma_{j}^{i}$ of a subsystem corresponding to a degenerate eigenvalue of $\mathbf{t}$, say $\beta$, will be of the form

$$
\Gamma_{j}^{i}(q, v)=\beta \delta_{j}^{i}+\Gamma_{j k}^{i}(q) v^{k},
$$

whereby the functions $\Gamma_{j k}^{i}(q)$ define a linear symmetric connection with zero curvature. From the theory of linear connections we then know that there exists a coordinate transformation which will make the transformed connection coefficients vanish. Such coordinates $Q^{i}$, in terms of which separability of the (sub-)system will finally be achieved, can be most easily found by constructing exact 1-forms which are "parallel". Explicitly, this amounts to constructing a number of 1-forms $\alpha^{j}$ satisfying

$$
\begin{aligned}
\alpha^{j} & =a_{i}^{j}(q) d q^{i}=d\left(Q^{j}(q)\right), \\
\widetilde{\nabla} \alpha^{j} & :=v^{\ell}\left(\frac{\partial a_{i}^{j}}{\partial q^{\ell}}-a_{k}^{j} \Gamma_{i \ell}^{k}\right) d q^{i}=0 .
\end{aligned}
$$

Observe, finally, as an interesting point of comparison for the explicit application treated in the next sections, that we know in the latter subcase how the right-hand sides $F^{i}$ of the decoupled equations will look like. Indeed, if $\Phi=\mu I$ and $\mathbf{t}=\beta I$ ( $\mu, \beta$ constant), then the decoupled form of the equations in question should be such that

$$
F^{i}=-\left(\mu+\beta^{2}\right) Q^{i}-2 \beta \dot{Q}^{i}+\gamma^{i},
$$

for some constants $\gamma^{i}$.

\section{Setting up a hard test for the theory}

Consider the following coupled non-linear system of second-order ordinary differential equations, depending on eighteen real parameters, (for notational convenience, coordinate indices are henceforth denoted as subscripts):

$$
\begin{aligned}
\ddot{q}_{1}= & b_{1} \dot{q}_{1}+a_{1} \dot{q}_{3}-s_{1} q_{1}+r_{1} q_{3}+a\left(q_{1}-q_{3}\right)^{2}, \\
\ddot{q}_{2}= & m_{1} \dot{q}_{1}^{2}+\dot{q}_{2}^{2}+m_{3} \dot{q}_{3}^{2}+n\left(2 \dot{q}_{1} \dot{q}_{2}-2 \dot{q}_{1} \dot{q}_{3}-\dot{q}_{2} \dot{q}_{3}\right)+ \\
& b_{2} \dot{q}_{2}+a_{2}\left(2 \dot{q}_{1}-\dot{q}_{3}\right)-s_{2} q_{2}+r_{2}\left(2 q_{1}-q_{3}\right), \\
\ddot{q}_{3}= & b_{3} \dot{q}_{3}+a_{3} \dot{q}_{1}-s_{3} q_{3}+r_{3} q_{1}+c q_{2}^{2}+b\left(q_{1}-q_{3}\right)^{2} .
\end{aligned}
$$


Before embarking on the long calculations, a few words are in order concerning the origin of this system. To be honest, we have made sure from the start that a subcase of known separability is built in. We have then added at least one non-linear coupling term which is intuitively expected to prohibit separability and, hence, its coefficient is expected to come out pretty soon as having to be zero. Next, we have replaced almost all numerical factors of the known separable case by as yet arbitrary parameters. Originally, the coefficient of $\dot{q}_{2}^{2}$ in the second equation then was an unspecified constant $m_{2}$. However, in a realistic situation one will normally try to eliminate redundant parameters by a suitable rescaling, and a preliminary analysis of the system revealed that one of the following parameters could be rescaled to 1 , namely: $a, m_{1}, m_{2}, m_{3}, n, c$, or $b$. We have selected $m_{2}$ as the most logical choice because all other constants are associated to coupling terms.

Applying the conditions of the separability theorem to the above system, we will now search for all regions in the 18-dimensional parameter space where the system admits a full decoupling. In agreement with the general strategy outlined in the previous section, we start by imposing the vanishing of the curvature tensor $R$. It immediately appears from this that there are only two admissible values for the parameter $n$, namely $n=2$ and $n=0$. In the next section we will pursue in some detail the separability analysis for the case where $n=2$ (case 1 ), whereas the case $n=0$ (case 2) will be treated more briefly in section 5 .

\section{Case $1: n=2$}

In this case it follows that $R$ identically vanishes if and only if

$$
m_{1}=4 ; \quad m_{3}=1 ; \quad b_{1}=\frac{1}{2} a_{3}-2 a_{1}+b_{3} ; \quad b_{2}=a_{2}-2 a_{1}+b_{3} .
$$

With these restrictions on the parameters, it turns out that also the condition $C_{\Phi}^{V}=0$ is already satisfied. Therefore, we can pass to the commutator condition $[\nabla \Phi, \Phi]=0$, which is definitely the most stringent among the algebraic equalities ensuing from the separability requirement.

The components of $[\nabla \Phi, \Phi]$, computed according to (10), are found to be linear in the velocities $v_{i}$, with coefficients that are themselves polynomials of degree three in the coordinates $q_{i}$. Inspection of the various independent monomials first of all reveals that, necessarily,

$$
c=0
$$

(c occurs, for instance, as coefficient of $q_{2}^{3} v_{3}$ in $[\nabla \Phi, \Phi]_{2}^{2}$ ). Next, the coefficient of $q_{3}^{3} v_{3}$ in $[\nabla \Phi, \Phi]_{1}^{1}$ imposes

$$
b=2 a .
$$


The parameter $a$ is then found as a factor in various of the remaining monomials. Hence, we are led to make a further subdivision according to whether $a$ is zero or not.

\subsection{Case 1.1: $a \neq 0$}

Taking into account all parameter conditions obtained so far, it follows from the coefficient of $q_{3}^{2}$ in $[\nabla \Phi, \Phi]_{1}^{1}$ that we must have

$$
a_{3}=-2 a_{1} \text {. }
$$

A careful analysis of the remaining conditions coming from $[\nabla \Phi, \Phi]=0$ shows that they are equivalent to the following three relations:

$$
r_{3}=-2 r_{1} \quad ; \quad s_{3}=s_{1}-3 r_{1} \quad ; \quad s_{2}=s_{1}-\left(r_{1}+r_{2}\right) .
$$

Summarizing, for $n=2$ and $a \neq 0$, the ten parameter relations given by (1519 ) are necessary and sufficient for having $R=0, C_{\Phi}^{V}=0$ and $[\nabla \Phi, \Phi]=0$. We next investigate diagonalizability of $\Phi$, the components of which are already reduced to:

$$
\begin{aligned}
\Phi_{1}^{1} & =\frac{1}{4}\left(8 a q_{3}-8 a q_{1}-7 a_{1}^{2}+6 a_{1} b_{3}-b_{3}^{2}+4 s_{1}\right), \\
\Phi_{2}^{1} & =0 \\
\Phi_{3}^{1} & =-\frac{1}{4}\left(8 a q_{3}-8 a q_{1}-3 a_{1}^{2}+2 a_{1} b_{3}+4 r_{1}\right), \\
\Phi_{1}^{2} & =-\frac{1}{2}\left[\left(4 s_{1}-4 r_{1}-4 r_{2}\right)\left(2 q_{1}+q_{2}-q_{3}\right)-4 a_{1} a_{2}+a_{2}^{2}+2 a_{2} b_{3}+4 r_{2}\right], \\
\Phi_{2}^{2} & =-\frac{1}{4}\left[\left(4 s_{1}-4 r_{1}-4 r_{2}\right)\left(2 q_{1}+q_{2}-q_{3}\right)+\right. \\
\Phi_{3}^{2} & =\frac{1}{4}\left[\left(4 s_{1}-4 r_{1}-4 r_{2}\right)\left(2 q_{1}+q_{2}-q_{3}\right)-4 a_{1} a_{2}+a_{2}^{2}+2 a_{2} b_{3}+4 r_{2}\right], \\
\Phi_{1}^{3} & =\frac{1}{2}\left(8 a q_{3}-8 a q_{1}-3 a_{1}^{2}+2 a_{1} b_{3}+4 r_{1}\right), \\
\Phi_{2}^{3} & =0, \\
\Phi_{3}^{3} & =-\frac{1}{4}\left(16 a a_{3}-16 a q_{1}-2 a_{1}^{2}+b_{3}^{2}+12 r_{1}-4 s_{1}\right) .
\end{aligned}
$$

We note, in particular, that the following relations hold:

$$
\Phi_{2}^{1}=\Phi_{2}^{3}=0, \Phi_{1}^{2}=-2 \Phi_{3}^{2}, \Phi_{1}^{3}=-2 \Phi_{3}^{1}, \quad \Phi_{1}^{1}-\Phi_{3}^{3}=-3 \Phi_{3}^{1} .
$$

Using these, the eigenvalues of $\Phi$ are easily found to be

$$
\begin{aligned}
\lambda_{1} & =\frac{1}{2}\left(\Phi_{1}^{1}+\Phi_{3}^{3}+\Phi_{3}^{1}\right)=\Phi_{3}^{3}-\Phi_{3}^{1}, \\
\lambda_{2} & =\Phi_{2}^{2}, \\
\lambda_{3} & =\frac{1}{2}\left(\Phi_{1}^{1}+\Phi_{3}^{3}-\Phi_{3}^{1}\right)=\Phi_{3}^{3}-2 \Phi_{3}^{1} .
\end{aligned}
$$


Note that, since $a \neq 0$, we clearly have $\lambda_{1} \neq \lambda_{3}$ and $\lambda_{1} \neq \lambda_{2}$. However, the degeneracy $\lambda_{2}=\lambda_{3}$ is still possible if both

$$
r_{2}=s_{1}-r_{1}
$$

and

$$
4\left(r_{1}-s_{1}\right)=a_{2}^{2}+2 a_{2} b_{3}-4 a_{1} a_{2}
$$

hold, which is in fact equivalent to $\Phi_{3}^{2}=0$. This forces us to make a further subdivision.

(i) Assume $\lambda_{2} \neq \lambda_{3}$ (i.e. $\Phi_{3}^{2} \neq 0$ ). $\Phi$ is now diagonalizable with three distinct eigenvalues, so we have complete separability. As outlined in the previous section, the construction of suitable coordinates in principle amounts to the simultaneous integration of the complementary eigendistributions of $\Phi$. In the present case, however, this is rather simple because the components of $\Phi$ are linear in the $q_{i}$ 's (and independent of the velocities), so that a linear coordinate transformation, $Q_{i}=a_{i j} q_{j}$ say, is expected to do the job. The procedure which will be adopted therefore, is to search for a regular constant matrix $A=\left(a_{i j}\right)$ such that

$$
A \Phi=\operatorname{diag}\left(\lambda_{1}, \lambda_{2}, \lambda_{3}\right) A .
$$

In view of the structure of $\Phi$, we may take an $A$ of the form

$$
A=\left(\begin{array}{lll}
1 & 0 & \alpha \\
\beta & 1 & \gamma \\
\delta & 0 & 1
\end{array}\right),
$$

and (22) then produces equations for the determination of $\alpha, \beta, \gamma$ and $\delta$. A solution is given by $\alpha=-1, \beta=2, \gamma=-1, \delta=-2$. Hence, decoupling should be achieved by the regular linear coordinate transformation

$$
Q_{1}=q_{1}-q_{3}, Q_{2}=2 q_{1}+q_{2}-q_{3}, Q_{3}=-2 q_{1}+q_{3},
$$

with inverse,

$$
q_{1}=Q_{1}-Q_{3}, q_{2}=Q_{2}+Q_{3}, q_{3}=-2 Q_{1}-Q_{3} .
$$

The transformed system (14) is indeed found to be:

$$
\begin{aligned}
& \ddot{Q}_{1}=\left(b_{3}-a_{1}\right) \dot{Q}_{1}-a Q_{1}^{2}+\left(2 r_{1}-s_{1}\right) Q_{1}, \\
& \ddot{Q}_{2}=\dot{Q}_{2}^{2}-\left(2 a_{1}-a_{2}-b_{3}\right) \dot{Q}_{2}-\left(s_{1}-r_{1}-r_{2}\right) Q_{2}, \\
& \ddot{Q}_{3}=\left(b_{3}-2 a_{1}\right) \dot{Q}_{3}+\left(r_{1}-s_{1}\right) Q_{3} .
\end{aligned}
$$

(ii) Assume $\lambda_{2}=\lambda_{3}$ (i.e. (20) and (21) hold or, equivalently, $\Phi_{3}^{2}=0$ ). It turns out that the same coordinate transformation as above diagonalizes $\Phi$ and also decouples the system, even though we are dealing with a degenerate 
eigenvalue. The least one expects then is that the additional conditions of the theorem, i.e. $C_{\mathbf{t}}^{V}=0$ and $\mathbf{t}$ diagonalizable, happen to be satisfied. One easily verifies that this is indeed the case. It is instructive to see that these conditions are nevertheless not redundant. To that end, observe that $\alpha=-1$, $\beta=\gamma=0$ and $\delta=-2$, provides another solution of (22) in this case. The corresponding transformation

$$
Q_{1}^{\prime}=q_{1}-q_{3}, Q_{2}^{\prime}=q_{2}, Q_{3}^{\prime}=-2 q_{1}+q_{3},
$$

diagonalizes $\Phi$, but leaves the subsystem $\left(Q_{2}^{\prime}, Q_{3}^{\prime}\right)$ internally coupled, so that a further transformation, coming from the analysis of the tension, is needed to achieve our goal.

REMARK: In analysing $\mathbf{t}$ for the subsystem in question, one comes across the possibility of a degenerate eigenvalue. This happens for $a_{2}=0$, which implies $r_{1}=s_{1}$ and $r_{2}=0$ (see (20) and (21)). Observe that the decoupled system (23) in this case contains

$$
\begin{aligned}
& \ddot{Q}_{2}=\dot{Q}_{2}^{2}+\left(b_{3}-2 a_{1}\right) \dot{Q}_{2}, \\
& \ddot{Q}_{3}=\left(b_{3}-2 a_{1}\right) \dot{Q}_{3} .
\end{aligned}
$$

The common factor $b_{3}-2 a_{1}$ is in agreement with the theory (see the factor $-2 \beta$ in (13)). The extra term $\dot{Q}_{2}^{2}$, on the other hand, would seem to contradict equation (13). However, under the present circumstances, the theory merely guarantees the existence of coordinates which will bring the "forces" in the form (13) (we have $\beta^{2}+\mu=0$ here) and of course does not preclude that separation may be achieved in other coordinates as well. To bring the above system in line with (13), it suffices to introduce the transformation $Q_{2}^{\prime}=$ $\exp \left(-Q_{2}\right)$ which can be obtained from the construction of a "parallel 1-form", as characterized by (12).

\subsection{Case 1.2: $a=0$}

In view of (17) we now also have that

$$
b=0 .
$$

Further analysis of the condition $[\nabla \Phi, \Phi]=0$ first reveals the following two additional restrictions on the parameters:

$$
r_{3}=2\left(r_{2}+s_{2}-s_{1}\right) ; s_{3}=r_{2}+s_{2}-2 r_{1} .
$$

The remaining (non-zero) components of $[\nabla \Phi, \Phi]$ now only contain constant terms, the vanishing of which entails just one extra condition which can be factorized as

$\left[r_{1} a_{3}+2 a_{1}\left(s_{1}-s_{2}-r_{2}\right)\right]\left[\left(a_{3}+4 a_{1}\right)\left(a_{3}-4 a_{1}+4 b_{3}\right)+16\left(r_{2}+2 r_{1}-s_{1}+s_{2}\right)\right]=0$. 
Accordingly, we identify two subcases distinguished by, respectively,

$$
r_{1} a_{3} \neq 2 a_{1}\left(r_{2}+s_{2}-s_{1}\right),
$$

and

$$
r_{1} a_{3}=2 a_{1}\left(r_{2}+s_{2}-s_{1}\right) .
$$

(i) If (26) holds, then (25) yields

$$
4 b_{3}\left(a_{3}+4 a_{1}\right)=16 a_{1}^{2}-a_{3}^{2}-16\left(r_{2}+2 r_{1}-s_{1}+s_{2}\right) .
$$

This can be regarded as a defining relation for $b_{3}$, since in this case we necessarily have $a_{3}+4 a_{1} \neq 0$. Indeed, (25) and (26) imply that $a_{3}+4 a_{1}=0$ if and only if $r_{2}=s_{1}-s_{2}-2 r_{1}$, and both relations together contradict (26).

We now turn to the diagonalizability condition of $\Phi$. Computing the components of $\Phi$ we observe that

$$
\Phi_{2}^{1}=\Phi_{2}^{3}=0, \Phi_{1}^{2}=-2 \Phi_{3}^{2}, \Phi_{1}^{3}=-4 \Phi_{3}^{1},
$$

which makes it easy to obtain the following eigenvalues:

$$
\begin{aligned}
& \lambda_{1}=\lambda_{3}=\frac{1}{2}\left(\Phi_{1}^{1}+\Phi_{3}^{3}\right), \\
& \lambda_{2}=\Phi_{2}^{2} .
\end{aligned}
$$

Further analysis reveals, however, that $\Phi$ in this case is not diagonalizable. Hence, no complete separation of (14) can be accomplished.

(ii) If (27) holds, the components of $\Phi$ verify the following relations:

$$
\Phi_{2}^{1}=\Phi_{2}^{3}=0, \Phi_{1}^{2}=-2 \Phi_{3}^{2}, \Phi_{1}^{1}-\Phi_{3}^{3}=\frac{1}{2} \Phi_{1}^{3}-2 \Phi_{3}^{1},
$$

and the eigenvalues of $\Phi$ now read

$$
\begin{aligned}
& \lambda_{1}=\frac{1}{2} \Phi_{1}^{3}+\Phi_{3}^{3}, \\
& \lambda_{2}=\Phi_{2}^{2}, \\
& \lambda_{3}=-2 \Phi_{3}^{1}+\Phi_{3}^{3} .
\end{aligned}
$$

Both $\lambda_{1}$ and $\lambda_{3}$ are found to be constant, whereas, if $s_{2} \neq 0, \lambda_{2}$ is not. The only possible degeneracy is then $\lambda_{1}=\lambda_{3}$. On the other hand, if $s_{2}=0$, then $\lambda_{2}$ is also constant, and various cases of degeneracy may occur. It turns out, however, that the nature of $\lambda_{2}$ does not have any effect on the further separability analysis of the case under consideration.

Using (28) and the explicit expressions for the components of $\Phi$, we find that

$$
\begin{aligned}
\lambda_{1}-\lambda_{3}= & \frac{1}{2} \Phi_{1}^{3}+2 \Phi_{3}^{1}= \\
& \frac{1}{16}\left[16 a_{1}^{2}-16 a_{1} b_{3}-a_{3}^{2}-4 a_{3} b_{3}-32 r_{1}-16\left(r_{2}+s_{2}-s_{1}\right)\right] .
\end{aligned}
$$


A straightforward analysis reveals that a solution of the matrix equation (22) is here given by

$$
A=\left(\begin{array}{ccc}
k_{1} & 0 & -k_{2} \\
2 & 1 & -1 \\
-2 & 0 & 1
\end{array}\right)
$$

where

$$
\begin{aligned}
& k_{1}=a_{3}^{2}-4 a_{1} a_{3}+4 a_{3} b_{3}+16\left(r_{2}+s_{2}-s_{1}\right), \\
& k_{2}=8 a_{1}^{2}-2 a_{1} a_{3}-8 a_{1} b_{3}-16 r_{1} .
\end{aligned}
$$

Since $\operatorname{det} A=k_{1}-2 k_{2}$, regularity requires $k_{1} \neq 2 k_{2}$. Observe at this point that we also have

$$
\lambda_{1}-\lambda_{3}=\frac{1}{16}\left(2 k_{2}-k_{1}\right) .
$$

This leads to the following discussion.

- If $\lambda_{1} \neq \lambda_{3}$ (i.e. $k_{1} \neq 2 k_{2}$ ), then $\Phi$ has three distinct eigenvalues and is diagonalized by the matrix $A$ of (29). By means of the corresponding transformation

$$
Q_{1}=k_{1} q_{1}-k_{2} q_{3}, Q_{2}=2 q_{1}+q_{2}-q_{3}, Q_{3}=-2 q_{1}+q_{3},
$$

the given system (14) effectively decouples and becomes:

$$
\begin{aligned}
& \ddot{Q}_{1}=\frac{1}{2}\left(a_{3}+2 b_{3}\right) \dot{Q}_{1}+\left(2 r_{1}-s_{1}\right) Q_{1}, \\
& \ddot{Q}_{2}=\dot{Q}_{2}^{2}+\left(a_{2}-2 a_{1}+b_{3}\right) \dot{Q}_{2}-s_{2} Q_{2}, \\
& \ddot{Q}_{3}=\left(b_{3}-2 a_{1}\right) \dot{Q}_{3}-\left(r_{2}+s_{2}\right) Q_{3} .
\end{aligned}
$$

- If $\lambda_{1}=\lambda_{3}$ (i.e. $k_{1}=2 k_{2}$ ), then the matrix (29) is no longer acceptable since $\operatorname{det} A=0$. For $k_{2} \neq 0$, it turns out that $\Phi$ is not diagonalizable and, hence, (14) cannot be completely separated. Assuming $k_{2}=0$, we also have $k_{1}\left(=2 k_{2}\right)=0$, and we can regard these conditions as defining relations for the parameters $r_{1}$ and $r_{2}$, namely

$$
\begin{aligned}
& r_{1}=\frac{1}{8}\left(4 a_{1}^{2}-a_{1} a_{3}-4 a_{1} b_{3}\right), \\
& r_{2}=s_{1}-s_{2}-\frac{1}{16}\left(a_{3}^{2}-4 a_{1} a_{3}+4 a_{3} b_{3}\right) .
\end{aligned}
$$

Note that these relations are compatible with the condition (27). We find that $\Phi$, which now has a degenerate eigenvalue, can be diagonalized by the linear coordinate transformation

$$
Q_{1}=q_{1}-q_{3}, Q_{2}=2 q_{1}+q_{2}-q_{3}, Q_{3}=-2 q_{1}+q_{3} .
$$


In the new coordinates, the system (14) reads:

$$
\begin{aligned}
\ddot{Q}_{1}= & \frac{1}{2}\left(a_{3}+2 b_{3}\right) \dot{Q}_{1}+\frac{1}{2}\left(2 a_{1}+a_{3}\right) \dot{Q}_{3}+\frac{1}{4}\left(4 a_{1}^{2}-a_{1} a_{3}-\right. \\
& \left.4 a_{1} b_{3}-4 s_{1}\right) Q_{1}+\frac{1}{16}\left(2 a_{1}+a_{3}\right)\left(4 a_{1}-a_{3}-4 b_{3}\right) Q_{3}, \\
\ddot{Q}_{2}= & \dot{Q}_{2}^{2}+\left(a_{2}-2 a_{1}+b_{3}\right) \dot{Q}_{2}-s_{2} Q_{2}, \\
\ddot{Q}_{3}= & \left(b_{3}-2 a_{1}\right) \dot{Q}_{3}-\left[s_{1}-\frac{1}{16}\left(a_{3}^{2}-4 a_{1} a_{3}+4 a_{3} b_{3}\right)\right] Q_{3}
\end{aligned}
$$

and, in a way which is consistent with the theory, is only partially decoupled. Note, however, that the system would be completely decoupled in case $a_{3}=-2 a_{1}$. Following the separability theorem, we next investigate the tension $\mathbf{t}$, associated with the $\left(Q_{1}, Q_{3}\right)$-subsystem, which is given by

$$
\mathbf{t}=\left(\begin{array}{cc}
\frac{1}{4}\left(a_{3}+2 b_{3}\right) & \frac{1}{4}\left(2 a_{1}+a_{3}\right) \\
0 & \frac{1}{2}\left(b_{3}-2 a_{1}\right)
\end{array}\right) .
$$

Clearly, $\mathbf{t}$ being constant, we have $C_{\mathbf{t}}^{V}=0$. The diagonal elements of $\mathbf{t}$ are its eigenvalues. If $a_{3}=-2 a_{1}, \mathbf{t}$ is diagonal and, as observed above, the system (30) is already completely decoupled. In the opposite case, $\mathbf{t}$ can only be diagonalized when its eigenvalues are different, i.e. provided that $a_{3} \neq-4 a_{1}$. The transformation

$$
Q_{1}^{\prime}=Q_{1}+\frac{2 a_{1}+a_{3}}{4 a_{1}+a_{3}} Q_{3} \quad, \quad Q_{3}^{\prime}=Q_{3},
$$

\begin{tabular}{|c|c|}
\hline \multicolumn{2}{|r|}{$c=0 \quad n=2$} \\
\hline \multicolumn{2}{|c|}{$\begin{array}{cc}m_{1}=4 & b_{1}=\frac{1}{2} a_{3}-2 a_{1}+b_{3} \\
m_{3}=1 & b_{2}=a_{2}-2 a_{1}+b_{3}\end{array}$} \\
\hline$a \neq 0$ & $a=0 \quad b=0$ \\
\hline$b=2 a$ & $s_{3}=s_{2}+r_{2}-2 r_{1}$ \\
\hline$a_{3}=-2 a_{1}$ & $r_{3}=2\left(r_{2}+s_{2}-s_{1}\right)$ \\
\hline$r_{3}=-2 r_{1}$ & - if $\left(a_{3}+4 a_{1}\right)\left(a_{3}-4 a_{1}+4 b_{3}\right)$ \\
\hline$s_{2}=s_{1}-\left(r_{1}+r_{2}\right)$ & $+8\left(r_{3}+4 r_{1}\right) \neq 0$ \\
\hline$s_{3}=s_{1}-3 r_{1}$ & then $r_{1} a_{3}=r_{3} a_{1}$ \\
\hline & $\begin{array}{c}\text { then } r_{1}=\frac{1}{8} a_{1}\left(4 a_{1}-a_{3}-4 b_{3}\right) \\
\star \text { else } a_{1}=0 \\
a_{3}=0 \\
r_{1}=0\end{array}$ \\
\hline
\end{tabular}

which does the diagonalization is then easily seen to decouple the system (30) as well.

This completes the study of case $1(n=2)$. As a summary, we list in the following table the parameter relations which in this case guarantee complete separability of the second-order system (14). 


\section{Case $2: n=0$}

The analysis of this case turns out to be much more involved than that of case 1. The parameter relations, ensuing from the separability conditions, are more complicated and there are also more subcases to be distinguished. Nevertheless, the general procedure being essentially the same as before, we will keep the treatment of case 2 as concise as possible.

First of all we find that, for $n=0, R$ vanishes if and only if

$$
m_{1}=0 ; m_{3}=0 ; a_{2}=0 ;
$$

and these conditions also guarantee the vanishing of $C_{\Phi}^{V}$. Among the numerous complicated expressions coming from the condition $[\nabla \Phi, \Phi]=0$, the requirements that are most easily detected read,

$$
r_{2}=0
$$

and $a c=0, b c=0$. For $c \neq 0$, we are led to various subcases for which it always appears, however, that $\Phi$ in the end is not diagonalizable. As in case 1 , we are thus forced to put

$$
c=0,
$$

which kills a lot of terms in the components of $[\nabla \Phi, \Phi]$. Nevertheless, there still remain some complicated parameter relations which, eventually, lead to the identification of the following three subcases:

$$
\begin{array}{ll}
\text { case } 2.1: & a=b=0 ; \\
\text { case } 2.2: & a=b \neq 0 ; \\
& b_{3}=b_{1}+a_{1}-a_{3} ; \\
& r_{3}=-r_{1}-\frac{1}{4}\left(a_{1}+a_{3}\right)\left(2 b_{1}+a_{1}-a_{3}\right) ; \\
& s_{3}=s_{1}-2 r_{1}-\frac{1}{4}\left(a_{1}+a_{3}\right)\left(2 b_{1}+a_{1}-a_{3}\right) ;
\end{array}
$$

case $2.3: a \neq b$

$$
\begin{aligned}
& b_{3}=b_{1}+a_{1}-a_{3} ; \\
& a a_{3}=-b a_{1} ; \\
& a r_{3}=-b r_{1} ; \\
& s_{3}=s_{1}+r_{3}-r_{1} .
\end{aligned}
$$

Case 2.2 is the easiest one to handle. It rapidly becomes clear that $\Phi$ has a non-diagonal Jordan normal form (with $\lambda_{1}=\lambda_{3}$ ) unless, among other things, we have $a=0$, which was excluded! The system therefore is not separable.

Case 2.3 is also quite tractable. It appears that the assumption $a \neq b$ ensures that the eigenvalues of $\Phi$ will all be different. Separability thus follows. Explicitly, a transformation which performs the separation is given by

$$
Q_{1}=-b q_{1}+a q_{2}, Q_{2}=q_{2}, Q_{3}=q_{3}-q_{1} .
$$


We then turn to the hard case 2.1.

PRELIMINARY REMARK: Under the current assumptions, the $q_{2}$-equation in (14) is already separated from the $\left(q_{1}, q_{3}\right)$-subsystem. This means that $\lambda_{2}=$ $\Phi_{2}^{2}$ will be an eigenvalue of $\Phi$ and that for further decoupling we have to worry about the complementary part of $\Phi$ only and, in particular, about possible degeneracy of $\lambda_{1}$ and $\lambda_{3}$ only. Moreover, in those cases where decoupling can be achieved, the corresponding coordinate transformation will merely affect $q_{1}$ and $q_{3}$ and we will systematically omit its trivial part $Q_{2}=q_{2}$.

In order for $[\nabla \Phi, \Phi]$ to be zero, it turns out that the remaining free parameters still have to verify three equations which, after some straightforward manipulations, can be cast in the form:

$$
\begin{aligned}
2 a_{1} a_{3}\left(b_{1}+\right. & \left.b_{3}\right)\left(s_{1}-s_{3}\right)+\left(a_{1} r_{3}+a_{3} r_{1}\right)\left[b_{1}^{2}-b_{3}^{2}+\right. \\
& \left.4\left(s_{1}-s_{3}\right)\right]+8 r_{1} r_{3}\left(b_{1}-b_{3}\right)=0 \\
2\left(a_{1} r_{3}-\right. & \left.a_{3} r_{1}\right)\left[\left(a_{1}+a_{3}\right)\left(b_{1}+b_{3}\right)+4\left(r_{1}+r_{3}\right)\right]+\left[b_{3}^{2}-b_{1}^{2}+\right. \\
& \left.4\left(s_{1}-s_{3}\right)\right]\left[\left(r_{1}-r_{3}\right)\left(b_{1}-b_{3}\right)+\left(a_{1}-a_{3}\right)\left(s_{1}-s_{3}\right)\right]=0, \\
2\left(a_{1} r_{3}-\right. & \left.a_{3} r_{1}\right)\left[\left(a_{1}-a_{3}\right)\left(b_{1}+b_{3}\right)+4\left(r_{1}-r_{3}\right)\right]+\left[b_{3}^{2}-b_{1}^{2}+\right. \\
& \left.4\left(s_{1}-s_{3}\right)\right]\left[\left(r_{1}+r_{3}\right)\left(b_{1}-b_{3}\right)+\left(a_{1}+a_{3}\right)\left(s_{1}-s_{3}\right)\right]=0 .
\end{aligned}
$$

A full discussion and analysis of these parameter conditions is a non-trivial enterprise. We briefly sketch how one may proceed.

First of all, observe that one may regard the equations (38) and (39) as a linear homogeneous system in the quantities $\rho=a_{1} r_{3}-a_{3} r_{1}$ and $\sigma=$ $b_{3}^{2}-b_{1}^{2}+4\left(s_{1}-s_{3}\right)$. Equation (37) then precisely expresses that the determinant of this system is zero. It is therefore not surprising that $\rho$ and $\sigma$ will play a key role in the subsequent discussion. From (38) and (39) one may easily infer that $\sigma=0$ necessarily implies $\rho=0$. Hence, if $\rho \neq 0$ we must have $\sigma \neq 0$ as well. In this case it remains a non-trivial problem to deduce some more tractable conditions from (37-39) and to use these for investigating the diagonalizability of $\Phi$. For all subcases which come into that discussion, however, $\Phi$ in the end turns out to be non-diagonalizable, so we omit reporting on more details.

Assume therefore that $\rho=0$. Adding and subtracting equations (38) and (39), and working out the left-hand side of (37), we obtain the equivalent system of equations

$$
\begin{aligned}
& {\left[2 a_{1}\left(b_{1}+b_{3}\right)+8 r_{1}\right]\left[\left(b_{1}-b_{3}\right) r_{3}+\left(s_{1}-s_{3}\right) a_{3}\right]=0,} \\
& \sigma\left[\left(b_{1}-b_{3}\right) r_{3}+\left(s_{1}-s_{3}\right) a_{3}\right]=0, \\
& \sigma\left[\left(b_{1}-b_{3}\right) r_{3}+\left(s_{1}-s_{3}\right) a_{1}\right]=0
\end{aligned}
$$

which, of course, has to be supplemented with the condition $\rho=0$. From (41) and (42), two subcases present themselves.

(I) Assume $\sigma \neq 0$.

The above system reduces to

$$
\left(b_{1}-b_{3}\right) r_{1}=\left(s_{3}-s_{1}\right) a_{1},
$$




$$
\left(b_{1}-b_{3}\right) r_{3}=\left(s_{3}-s_{1}\right) a_{3},
$$

and again gives rise to two subcases.

- If $b_{1} \neq b_{3}$, then (43) and (44) can be used as defining relations for $r_{1}$ and $r_{3}$ (and note that the resulting expressions are compatible with the assumption $\rho=0$ ). Computing $\Phi$ we find, listing only the part of interest (recall that the $q_{2}$-equation is already separated from the rest):

$$
\begin{aligned}
& \Phi_{1}^{1}=-\frac{1}{4}\left(a_{1} a_{3}+b_{1}^{2}-4 s_{1}\right) \quad, \quad \Phi_{3}^{1}=\frac{-a_{1} \sigma}{4\left(b_{1}-b_{3}\right)} ; \\
& \Phi_{1}^{3}=\frac{-a_{3} \sigma}{4\left(b_{1}-b_{3}\right)} \quad, \quad \Phi_{3}^{3}=-\frac{1}{4}\left(a_{1} a_{3}+b_{3}^{2}-4 s_{3}\right) .
\end{aligned}
$$

The remaining eigenvalues are obtained as,

$$
\lambda_{1,3}=\frac{4\left(b_{1}-b_{3}\right)\left(\Phi_{1}^{1}+\Phi_{3}^{3}\right) \pm \sigma \sqrt{4 a_{1} a_{3}+\left(b_{1}-b_{3}\right)^{2}}}{8\left(b_{1}-b_{3}\right)} .
$$

Clearly, for these to be real one should have that $4 a_{1} a_{3}+\left(b_{1}-b_{3}\right)^{2} \geq 0$. In case the equality holds and, hence, $\lambda_{1}=\lambda_{3}$, one can prove that $\Phi$ is not diagonalizable. We thus have to impose the additional restriction

$$
D=4 a_{1} a_{3}+\left(b_{1}-b_{3}\right)^{2}>0,
$$

which then ensures that $\Phi$ must be diagonalizable by a linear coordinate transformation, and the system will decouple. In constructing this transformation, i.e. in identifying a suitable regular matrix $A$ verifying (22), we again have to distinguish between several cases. Omitting here also the irrelevant middle row and middle column of $A$, the results we obtained read as follows.

If $\sqrt{D} \neq b_{1}-b_{3}$, a solution is given by

$$
A=\left(\begin{array}{cc}
\frac{1}{2}\left(b_{1}-b_{3}-\sqrt{D}\right) & a_{1} \\
a_{3} & -\frac{1}{2}\left(b_{1}-b_{3}-\sqrt{D}\right)
\end{array}\right) .
$$

If $\sqrt{D}=b_{1}-b_{3}(\neq 0)$, with $a_{3}=0$,

$$
A=\left(\begin{array}{cc}
0 & 1 \\
\frac{1}{2}\left(b_{1}-b_{3}\right) & \frac{1}{2} a_{1}
\end{array}\right) .
$$

Finally, if $\sqrt{D}=b_{1}-b_{3}(\neq 0)$, with $a_{1}=0$,

$$
A=\left(\begin{array}{cc}
-\frac{1}{2} a_{3} & \frac{1}{2}\left(b_{1}-b_{3}\right) \\
1 & 0
\end{array}\right)
$$


- Next, assume $b_{1}=b_{3}$. Since $\sigma \neq 0$ it follows that $s_{1} \neq s_{3}$ and, hence, (43) and (44) yield

$$
a_{1}=a_{3}=0 .
$$

The remaining eigenvalues of $\Phi$ are now given by

$$
\lambda_{1,3}=\frac{1}{2}\left[\Phi_{1}^{1}+\Phi_{3}^{3} \pm \sqrt{D^{\prime}}\right]
$$

with $D^{\prime}=4 r_{1} r_{3}+\left(s_{3}-s_{1}\right)^{2}$. For $D^{\prime}=0$, i.e. for $\lambda_{1}=\lambda_{3}$, it is found that $\Phi$ is not diagonalizable and thus we have to require

$$
4 r_{1} r_{3}+\left(s_{3}-s_{1}\right)^{2}>0,
$$

which will ensure complete decoupling. For the Jacobian matrix $A$ of a linear transformation which accomplishes the decoupling we obtain this time:

for $\sqrt{D^{\prime}} \neq s_{1}-s_{3}$,

$$
A=\left(\begin{array}{cc}
-2 r_{3} & \sqrt{D^{\prime}}+s_{3}-s_{1} \\
\sqrt{D^{\prime}}+s_{3}-s_{1} & 2 r_{1}
\end{array}\right)
$$

for $\sqrt{D^{\prime}}=s_{1}-s_{3}(\neq 0)$, with $r_{3}=0$,

$$
A=\left(\begin{array}{cc}
s_{3}-s_{1} & r_{1} \\
0 & 1
\end{array}\right)
$$

for $\sqrt{D^{\prime}}=s_{1}-s_{3}(\neq 0)$, with $r_{1}=0$,

$$
A=\left(\begin{array}{cc}
1 & 0 \\
-r_{3} & s_{3}-s_{1}
\end{array}\right) \text {. }
$$

This completes the study of subcase (I) of case 2.1.

(II) Assume now $\sigma=\rho=0$.

Conditions (41) and (42) are satisfied and we are left with the restriction (40). Without entering the details of the discussion, we give an overview of the various possibilities one encounters in this case.

- Let $b_{1}=b_{3}$. In view of $\sigma=0$, we then also have $s_{1}=s_{3}$. For $\Phi$ to admit real eigenvalues we must have $a_{1} a_{3} \geq 0$.

First, assume $a_{1} a_{3}>0$. Then, if $r_{1} \neq-\frac{1}{2} a_{1} b_{1}, \Phi$ is diagonalizable with $\lambda_{1} \neq \lambda_{3}$, and decoupling can be accomplished by

$$
A=\left(\begin{array}{cc}
\frac{1}{2}\left(a_{1}+a_{3}\right)\left(a_{3} b_{1}+2 r_{3}\right) & -\sqrt{a_{1} a_{3}}\left[\frac{1}{2}\left(a_{1}+a_{3}\right) b_{1}+r_{1}+r_{3}\right] \\
\sqrt{a_{1} a_{3}}\left[\frac{1}{2}\left(a_{1}+a_{3}\right) b_{1}+r_{1}+r_{3}\right] & \frac{1}{2}\left(a_{1}+a_{3}\right)\left(a_{1} b_{1}+2 r_{1}\right)
\end{array}\right) .
$$


- If $r_{1}=-\frac{1}{2} a_{1} b_{1}$ (and thus, in view of $\rho=0, r_{3}=-\frac{1}{2} a_{3} b_{1}$ ), then $\Phi$ is already in diagonal form with $\lambda_{1}=\lambda_{3}$. The system (14) is not yet completely decoupled. The tension $\mathbf{t}$ corresponding to the $\left(q_{1}, q_{3}\right)$-subsystem, reads

$$
\mathbf{t}=\left(\begin{array}{cc}
\frac{b_{1}}{2} & \frac{a_{1}}{2} \\
\frac{a_{3}}{2} & \frac{b_{1}}{2}
\end{array}\right)
$$

and, obviously, verifies $C_{\mathbf{t}}^{V}=0$. Moreover, $\mathbf{t}$ can be brought into diagonal form by means of the transformation

$$
Q_{1}=a_{3} q_{1}+\sqrt{a_{1} a_{3}} q_{3}, Q_{3}=a_{3} q_{1}-\sqrt{a_{1} a_{3}} q_{3},
$$

which establishes complete decoupling of (14).

Next, assume $a_{1} a_{3}=0$. The analysis now shows that the only favourable cases are those where $a_{1}=a_{3}=0$ and either $r_{1} r_{3}>0$ or $r_{1}=r_{3}=0$. In the latter case, the system (14) is already decoupled as it stands. In the former case, $\Phi$ is diagonalizable with $\lambda_{1} \neq \lambda_{3}$ and a transformation which diagonalizes $\Phi$ and decouples (14), reads

$$
Q_{1}=r_{3} q_{1}-\sqrt{r_{1} r_{3}} q_{3}, Q_{3}=r_{3} q_{1}+\sqrt{r_{1} r_{3}} q_{3} .
$$

- Let $b_{1} \neq b_{3}$. Further analysis of the parameter conditions, in particular of equation (40), gives rise to the following three possibilities: (i) $a_{3}=r_{3}=$ 0 ; (ii) $a_{1}=r_{1}=0$; (iii) $a_{1} a_{3} \neq 0, r_{1}=-\frac{1}{4} a_{1}\left(b_{1}+b_{3}\right), r_{3}=-\frac{1}{4} a_{3}\left(b_{1}+b_{3}\right)$.

(i) If $a_{3}=r_{3}=0$, then $\Phi$ is already in Jordan normal form with $\lambda_{1}=\lambda_{3}$, and will be diagonal iff $r_{1}=-\frac{1}{4} a_{1}\left(b_{1}+b_{3}\right)$. The $q_{1}$-equation in (14) then still has a $q_{3}$-dependence. The tension $\mathbf{t}$ of the $\left(q_{1}, q_{3}\right)$-subsystem satisfies $C_{\mathbf{t}}^{V}=0$ and has two distinct (real) eigenvalues. Hence, $\mathbf{t}$ can be diagonalized and a further decoupling of the system can be carried through by means of the transformation

$$
Q_{1}=\frac{1}{2}\left(b_{1}-b_{3}\right) q_{1}+\frac{1}{2} a_{1} q_{3}, Q_{3}=q_{3} .
$$

(ii) If $a_{1}=r_{1}=0$, then we are in a situation similar to the previous one. $\Phi$ will be diagonal iff $r_{3}=-\frac{1}{4} a_{3}\left(b_{1}+b_{3}\right)$ and, this time, it is the $q_{3}$-equation which is not yet decoupled. The tension of the $\left(q_{1}, q_{3}\right)$ subsystem verifies the conditions of the separability theorem, and complete decoupling of (14) is accomplished by the transformation

$$
Q_{1}=q_{1}, Q_{3}=-\frac{1}{2} a_{3} q_{1}+\frac{1}{2}\left(b_{1}-b_{3}\right) q_{3} .
$$

(iii) If $a_{1} a_{3} \neq 0, r_{1}=-\frac{1}{4} a_{1}\left(b_{1}+b_{3}\right)$ and $r_{3}=-\frac{1}{4} a_{3}\left(b_{1}+b_{3}\right)$, then $\Phi$ is diagonal with $\lambda_{1}=\lambda_{3}$, but again the system (14) is not yet completely decoupled. Diagonalizability of the tension now requires

$$
4 a_{1} a_{3}+\left(b_{1}-b_{3}\right)^{2}>0 .
$$


An appropriate transformation leading to complete decoupling of (14), is then given by

$$
\begin{aligned}
& Q_{1}=a_{3} q_{1}+\frac{1}{2}\left(\sqrt{4 a_{1} a_{3}+\left(b_{1}-b_{3}\right)^{2}}+b_{3}-b_{1}\right) q_{1}, \\
& Q_{3}=a_{3} q_{1}-\frac{1}{2}\left(\sqrt{4 a_{1} a_{3}+\left(b_{1}-b_{3}\right)^{2}}+b_{3}-b_{1}\right) q_{3} .
\end{aligned}
$$

This completes the study of case 2.1 and, at the same time, concludes the separability analysis of the second-order system (14).

In the table below we give an overview of the parameter relations which guarantee complete separability in the case $n=0$. In interpreting this classification, one should keep in mind that whenever we are in a situation where the separability conditions are verified, the actual construction of the decoupling transformation may still lead to different subcases. As a result, some of the favourable cases listed below, encompass two or more of the subcases encountered in the above analysis.

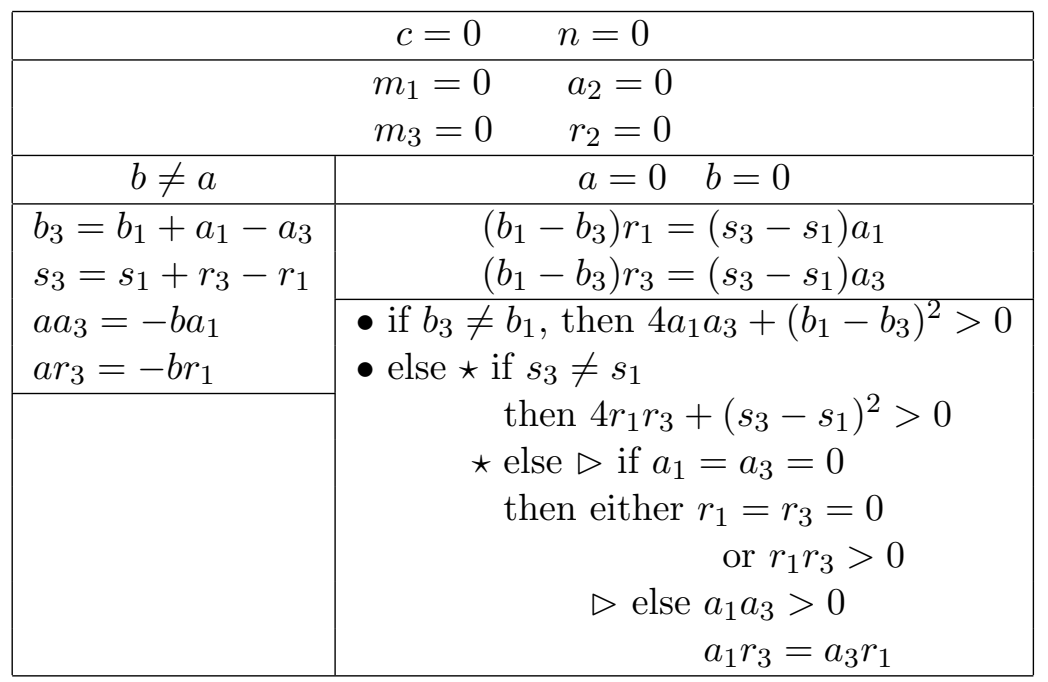

\section{Conclusions}

The separability theory developed in [5] has been put to a serious test by means of a system of three coupled, nonlinear second-order ordinary differential equations, depending on eighteen parameters. In particular, we have succeeded in identifying all domains in the parameter space where the system admits a full decoupling. Moreover, for each favourable case we have also constructed the explicit form of the transformation which accomplishes the decoupling. Although, the given system may look rather artificial at the outset, for the purpose of testing the theory it turned out to be extremely useful. 
Apart from providing us with a highly non-trivial example, it has allowed us to illustrate the relevance and role of all conditions entering the separability theorem.

Acknowledgement. Support from the Belgian National Fund for Scientific Research is gratefully acknowledged.

\section{References}

[1] C. Ferrario, G. Lo Vecchio, G. Marmo and G. Morandi, Separability of completely integrable systems admitting alternative Lagrangian descriptions, Lett. Math. Phys. 1985, V.9, 141-148.

[2] C. Ferrario, G. Lo Vecchio, G. Marmo and G. Morandi, A separability theorem for dynamical systems admitting alternative Lagrangians, $J$. Phys. A: Math. Gen. 1987, V.20, 3225-3236.

[3] M. Kossowski and G. Thompson, Submersive second-order differential equations, Math. Proc. Camb. Phil. Soc. 1991, V.110, 207-224.

[4] E. Martínez, J.F. Cariñena and W. Sarlet, Derivations of differential forms along the tangent bundle projection. Part II, Diff. Geometry and its Applications. 1993, V.3, 1-29.

[5] E. Martínez, J.F. Cariñena and W. Sarlet, Geometric characterization of separable second-order differential equations, Math. Proc. Camb. Phil. Soc. 1993, V.113, 205-224.

[6] W. Sarlet and E. Martínez, Separability of second-order differential equations, in: D. Bainov and V. Covachev, eds., Differential Equations, Proc. Second Colloquium 1991, Plovdiv, Bulgaria (World Scientific, 1992), 191203. 